\title{
Spontaneous Perforation of an Acalculous Hydropic Gallbladder in a Diabetic Patient With Neuropathy: An Underdiagnosed Entity
}

\author{
Shivantha Amarnath ${ }^{\mathrm{a}, \mathrm{c}}$, Abhishek Datta Polavarapu ${ }^{\mathrm{b}}$, Vivek Gumaste ${ }^{\mathrm{b}}$
}

\begin{abstract}
Gallbladder hydrops (HGB) is often under-diagnosed due to the various diagnostic criteria used throughout the globe. It can be described as a progressive increase in the volume of the gallbladder in the absence of inflammation, or bacterial infection. The underlying mechanism involves biliary ductal obstruction due to gallstones. We describe a unique case of a diabetic patient who presented with symptoms of viral gastroenteritis and was incidentally found to have a non-traumatic spontaneous rupture of a hydropic gallbladder which resulted in septic shock and eventual death of the patient. We present this case together with a comprehensive literature review on why diabetic patients are at high risk of HGB and subsequent spontaneous perforation compared to the general population.
\end{abstract}

Keywords: Gallbladder hydrops; Cholecystomegaly; Gallbladder perforation; Gallbladder rupture

\section{Introduction}

Gallbladder hydrops (HGB) is a rare entity that attributes to $<5 \%$ of all cases of gallbladder diseases. It is under-diagnosed due to the various diagnostic criteria used throughout the globe. It can be defined as a progressive increase in the volume of the gallbladder in the absence of any underlying evidence of inflammation, bacterial infection, or ductal abnormalities. The most common etiology is an obstructed gallstone. This leads to the production of mucoid or watery material from the mucosal wall with subsequent resorption of bile. Symptoms of HGB are often vague and may include epigastric pain, dys-

Manuscript submitted September 17, 2019, accepted October 11, 2019

aDepartment of Internal Medicine, Staten Island University Hospital, Northwell Health, Staten Island, NY, USA

bDepartment of Gastroenterology, Staten Island University Hospital, Northwell Health, Staten Island, NY, USA

${ }^{\mathrm{c} C o r r e s p o n d i n g ~ A u t h o r: ~ S h i v a n t h a ~ A m a r n a t h, ~ D e p a r t m e n t ~ o f ~ I n t e r n a l ~ M e d i-~}$ cine, Staten Island University Hospital, 70 Hancock Street, Staten Island, NY 10305, USA. Email: samarnath@northwell.edu

doi: https://doi.org/10.14740/gr1226 pepsia and chronic backache. Surgery is the ultimate treatment for HGB. However, conservative management is initially pursued in high-risk patients as it is expected that the passage of the obstructed gallstone may decompress the gallbladder, thus avoiding any surgical complications.

Gallbladder disorders in diabetic patients are often clinically silent and can present suddenly with catastrophic complications requiring emergency surgery. Current literature states that the duration of diabetes mellitus has a positive correlation with the prevalence of gallbladder dysfunction and subsequent gallbladder diseases. As the global prevalence of diabetes mellitus is projected to increase from 366 million in 2011 to 552 million by 2030 , it is possible to theorize that diabetic patients will contribute to a broader distribution of patients suffering from various gallbladder diseases [1]. Diabetic patients may be more prone to have HGB due to the progressive increase in gallbladder volume even in the absence of an overt biliary ductal obstruction. We describe a unique case of a diabetic patient who presented with symptoms of viral gastroenteritis and was incidentally found to have a non-traumatic spontaneous rupture of a hydropic gallbladder. We present this case along with an up-to-date literature review on the mechanisms involved in the atypical development of HGB in diabetic patients, especially those with neuropathy. Additionally, we hope to raise awareness amongst clinicians of this entity and propose that these patients may benefit from an early screening of gallbladder dysfunction.

\section{Case Report}

A 78-year-old man with history of triple coronary artery bypass on aspirin, heart failure with reduced ejection fraction of $30 \%$ with placement of an automated implanted cardioverter, noninsulin dependent diabetes mellitus complicated by neuropathy presented with 1-day history of dull epigastric abdominal pain associated with non-bilious, non-bloody vomiting along with watery diarrhea. The pain worsened with consumption of food. History dates back to 1 week prior, when his grandson developed similar symptoms and was hospitalized for viral gastroenteritis. Our patient, who lived with his grandson, also developed viral symptoms 3 days before the onset of vomiting and diarrhea. He denied any recent fevers, red blood per rectum, dark urine, pruritus or weight loss. 

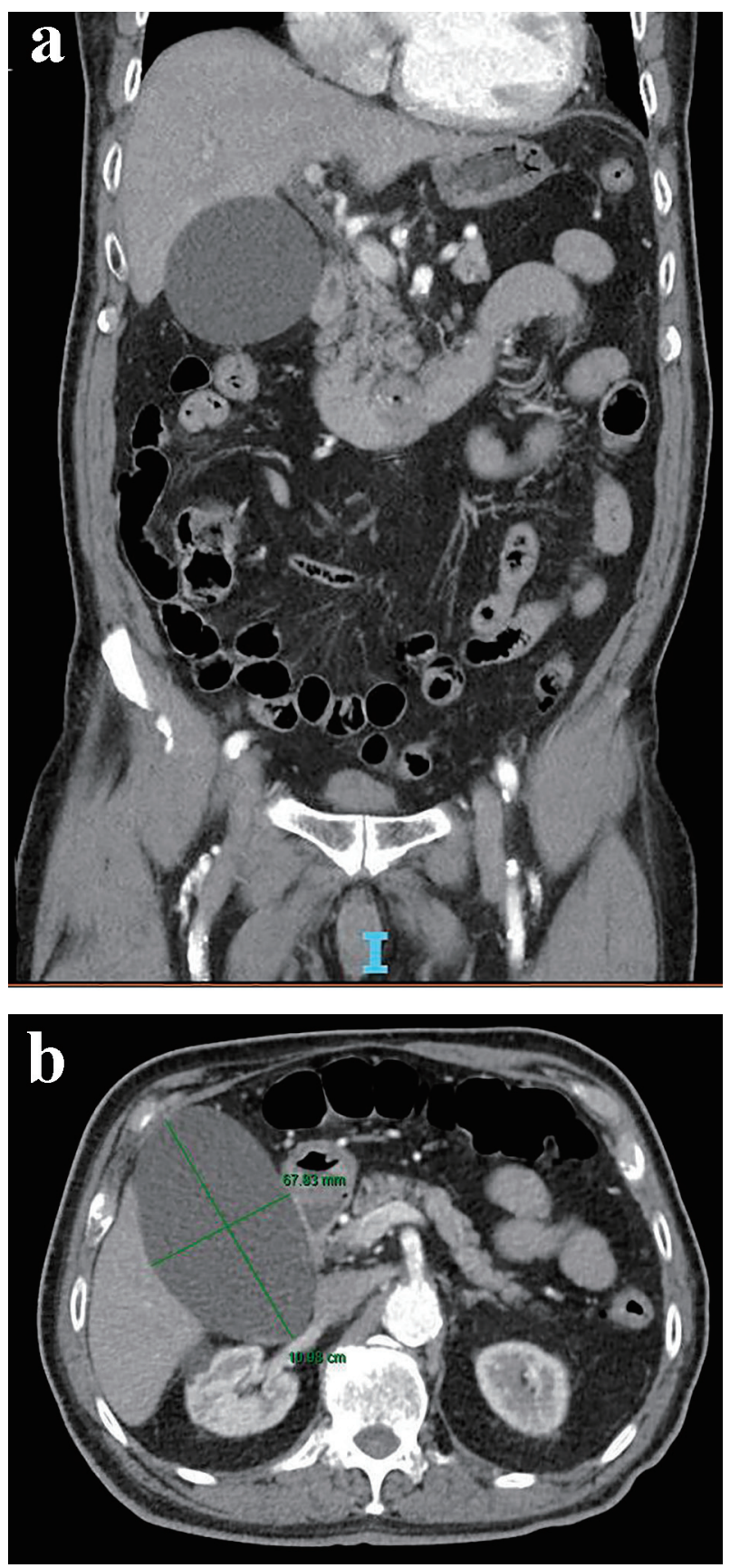

Figure 1. Coronal (a) and axial view (b) of a contrast-enhanced abdominal CT scan showing an acalculous hydropic gallbladder. CT: computed tomography.

In the emergency room, the patient was afebrile and did not meet sepsis criteria. Laboratory findings including lactate, amylase, lipase and hepatic function panel were all unremarkable. He underwent computed tomography (CT) of the abdomen with intravenous (IV) contrast, which incidentally demonstrated a hydropic gallbladder measuring $6.7 \times 11 \mathrm{~cm}$ without evidence of cholecystitis. There was no evidence of hepatic biliary ductal dilation, and common bile duct measured $0.5 \mathrm{~cm}$ (Fig. 1). Subsequently, a right upper quadrant ultrasound of

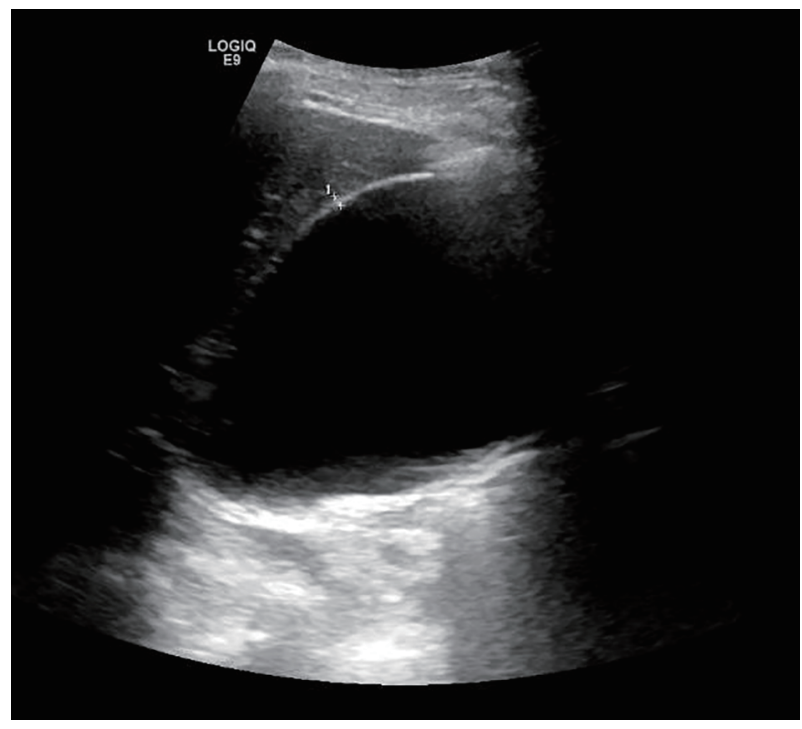

Figure 2. Ultrasound imaging of an acalculous hydropic gallbladder with demonstration of gallbladder sludge and without evidence of gallbladder wall thickening or pericholecystic fluid.

the abdomen demonstrated gallbladder sludge without any evidence of gallbladder wall thickening or peri-cholecystic fluid (Fig. 2). Sonographic Murphy's sign was negative. Surgery was consulted for evaluation of the hydropic gallbladder and recommended conservative management with possible cholecystectomy on an outpatient basis considering his high risk based on American Society of Anesthesiologist (ASA) physical status classification score of III. The patient was admitted with a diagnosis of viral gastroenteritis and began supportive therapy.

Five hours later, patient was febrile and endorsed worsening epigastric pain, now with radiation to the right flank. A hepatobiliary iminodiacetic acid (HIDA) scan demonstrated patency of the cystic duct by visualization of the gallbladder at $15 \mathrm{~min}$ post-injection of contrast. Strikingly, at the 45 -min mark, there was evidence of free bile within the right peritoneal cavity that increased through $4 \mathrm{~h}$ post-imaging, which suggested early evidence of gall bladder rupture (Fig. 3). The patient was placed on IV antibiotics and hydration. Surgery was promptly re-consulted. A repeat CT of the abdomen was obtained to evaluate the source of the bile leak and demonstrated interval decrease in size of the gall bladder with perihepatic fluid tracking along the right paracolic gutter and into the pelvis. The site of the biliary leak was not discernible (Fig. 4). The patient was septic due to possible peritonitis from rupture of the contents of the gallbladder. He underwent urgent subtotal cholecystectomy via diagnostic laparoscopy of the perforated gallbladder along with biliary stent placement. The surgeons found evidence of significant inflammation surrounding the gallbladder along with biliary peritonitis. Evaluation of the resected gallbladder did not reveal any evidence of stones and biopsy revealed that the serosa was congested with foci of yellow exudate along with multiple depressions in the mucosal wall. The wall was only $0.1 \mathrm{~mm}$ thick. Patient eventually required intubation and IV pressure support therapy. His course 


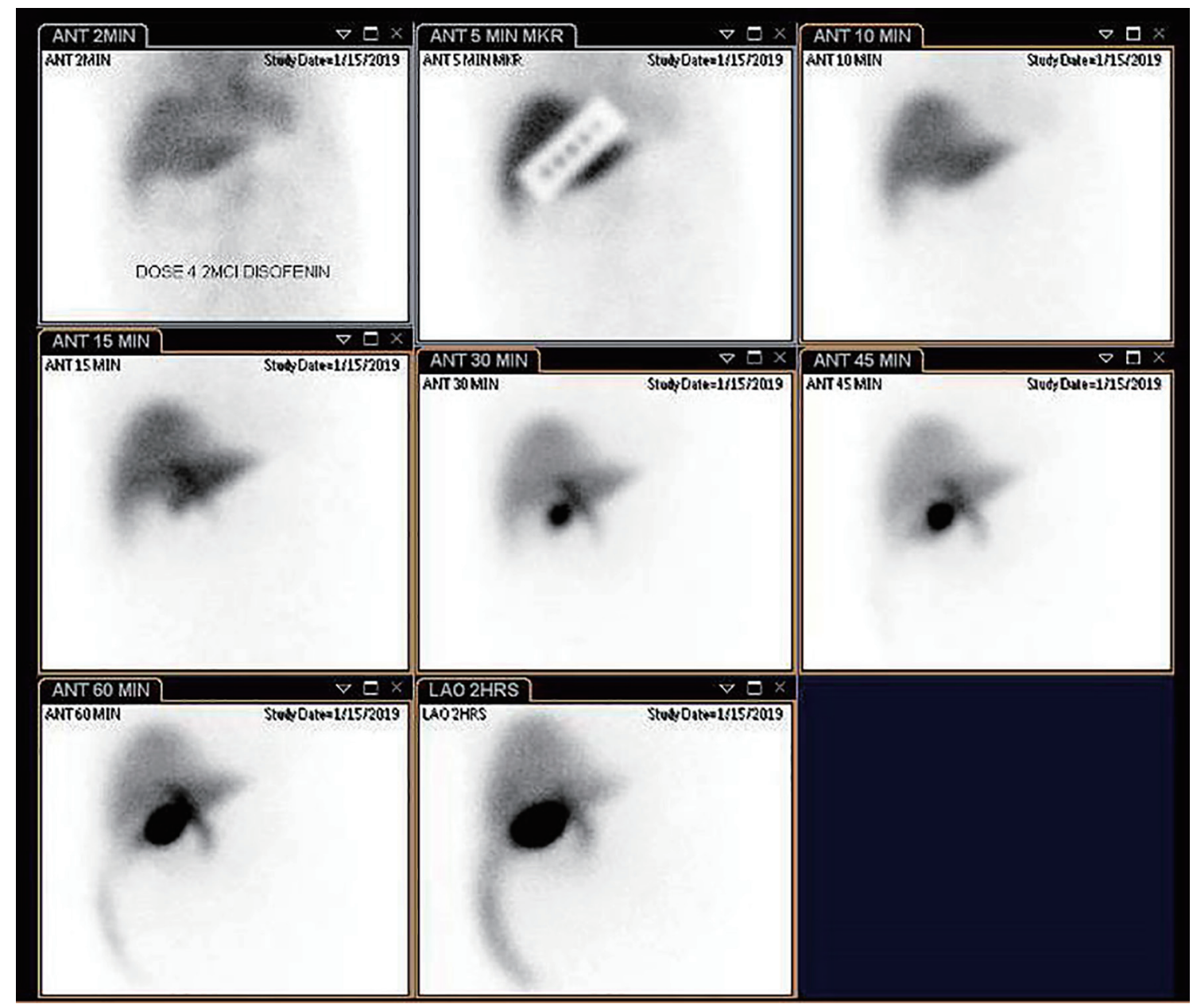

Figure 3. Hepatobiliary iminodiacetic acid (HIDA) scan with visualization of the gallbladder within 15 min post-injection of bright nuclear material. There is also early evidence of biliary leak into the right peritoneal cavity at 45 min which increases up to $4 \mathrm{~h}$ post-contrast admission, which suggests a perforated gallbladder.

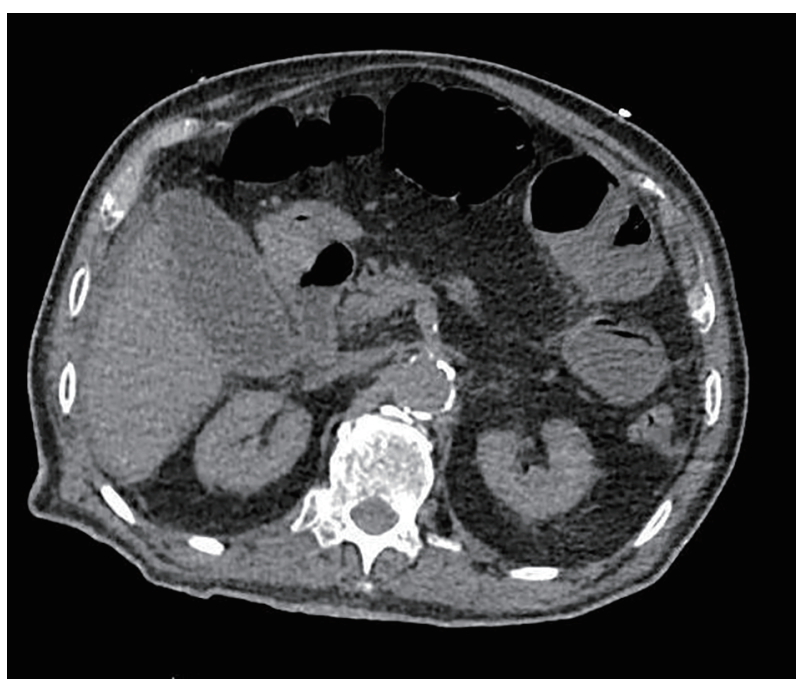

Figure 4. Axial view of a contrast-enhanced CT of the abdomen demonstrating a decompressed gallbladder and biliary leakage. CT: computed tomography. was further complicated by multi-organ failure, and he expired on day 6.

\section{Discussion}

Gallbladder diseases are categorized into acute and acalculous, and the latter is further subdivided into hydrops and cholecystitis [2]. HGB is a rare entity and attributes to roughly $3 \%$ of all gallbladder diseases [3]. Absence of inflammation is vital for the diagnosis of HGB [2]. At early stages of an HGB, the clinical symptoms can be vague but with prolonged stasis of the biliary contents, bacterial colonization can occur leading to presentation of cholecystitis [4]. Long-term complications of HGB can include malignant conversion [4]. Several studies in the literature have uncovered that the risk of gallbladder disease overall is increased in those with diabetes mellitus [5-7]. A recent meta-analysis found that the risk of gallbladder diseases is increased by $56 \%$ amongst diabetic patients compared to the general population [5]. Chronicity of diabetes itself has a positive correlation for this increased prevalence and progres- 
sive increase in gallbladder volume leading to hydrops [7].

Normal gallbladder volume is $8 \times 4 \mathrm{~cm}$ when fully distended, and in HGB, the anterior-posterior diameter is greater than $5 \mathrm{~cm}$, or enlargement is greater than $4 \times 10 \mathrm{~cm}[4,8]$. $\mathrm{HGB}$ is diagnosed incidentally on routine imaging or at the time of cholecystectomy [9]. Cholecystomegaly occurs most commonly due to a longstanding obstruction of the biliary tree, and this leads to the slow resorption of bile with continued production of mucoid content from the gallbladder mucosa causing dilatation [8]. Obstruction of the cystic duct due to cholelithiasis is the most common cause. However, strictures, parasites and external compression by a tumor or liver abscess can also contribute to an HGB [4, 9]. It should also be noted that risk factors of cholecystitis especially, rapid weight loss, prolonged total parenteral nutrition, dyslipidemia can lead to the formation of an HGB [9]. The wall of an HGB can progress to become thin and dilated with subsequent ischemia and risk of perforation and sepsis.

Gallbladder dysmotility plays a crucial role in biliary stasis and stone formation and in diabetic patients, this is chiefly due to autonomic dysfunction and reduced hormonal responses. Diabetic autonomic neuropathy manifests as esophagopathy, gastroparesis, enteropathy and biliary tract dysfunction and even HGB without any evidence of mechanical obstruction $[4,7]$. Autonomic neuropathy itself leads to impaired gallbladder emptying, and this can be demonstrated by the impaired gallbladder ejection fraction amongst those with diabetes. One study also found that the gallbladder ejection fraction remained suppressed even after achieving euglycemia for a duration of 3 - 6 months $[7,10]$. Diabetic patients with neuropathy can also have increased fasting gallbladder volume and diminished gallbladder contractility. These two factors progressively get worse with the course of diabetes and can be used as markers of biliary dysfunction and stasis $[6,11]$. Biliary saturation index can also be significantly increased amongst diabetic patients [5].

The smooth muscle cells of the gallbladder wall of diabetic patients have reduced sensitivity to cholecystokinin (CCK), and it is proposed that there are reduced numbers of CCK receptors found within these cells in diabetic patients [5]. Hyperglycemia itself can delay gastric emptying in the absence of autonomic neuropathy, via glycation of surface proteins, nucleic acids and lipids of the gallbladder wall affecting permeation and response to CCK [10]. The rate of release of CCK is dependent on the release of chyme. The transit of chyme is slow in those with gastroparesis, and CCK is found at low levels, which further contributes to the impaired sensitivity of the gallbladder wall to CCK [4]. It is well known that triglycerides play a key role in the formation of gallstones. Certain studies have found that low-density lipoprotein (LDL) can increase gallbladder volume in diabetes; however, another study did not find any improvement in gallbladder emptying with statin therapy in the diabetic population [12].

Gallbladder perforation (GBP) occurs in $2-15 \%$ cases of acute cholecystitis and is mostly triggered by acute inflammation of biliary contents. HGB may carry a high risk of perforation, but we did not come across any data in the literature documenting this. It may be due to the fact that HGB is mostly encountered during surgery and cholecystectomy reduces the risk of spontaneous rupture. Rarely, sonographic imaging of the gallbladder may reveal a "hole sign" defect in the gallbladder wall indicating GBP [13]. Other non-specific signs of GBP can include wall thickening $>0.3 \mathrm{~cm}$, gallbladder distention $>4$ $\mathrm{cm}$ (mimicking findings of acute uncomplicated cholecystitis), evidence of coarse intra-cholecystic echogenic debris and biliary ductal dilation [14]. CT of the abdomen and pelvis is more likely to establish a diagnosis. It should be emphasized that patients with atherosclerotic heart disease and diabetes mellitus are more prone to GBP compared to the general population [13]. Also, it is possible to theorize that in our patient, physical and sonographic evaluation of the gallbladder using the Murphy's sign may have caused further stress on the hydropic gallbladder and possibly may have played a role in its perforation.

Based on the risk factors discussed above, we propose that chronic diabetic patients may benefit from an early screening of gallbladder dysfunction via the assessment of impaired fasting gallbladder volume, postprandial gallbladder emptying, gallbladder sludge and stone formation $[4,15]$. It is imperative to appreciate that the size of the gallbladder will not decrease in those with diabetes, especially in the absence of mechanical obstruction. Thus, early diagnosis and surgical intervention at an opportune moment is preferable in order to reduce longterm morbidity and mortality [4].

\section{Conclusion}

HGB is a rare manifestation in the realm of gallbladder diseases, and patients with diabetes are more predisposed to its development. Clinical presentation is often non-specific and can include dyspepsia and upper back pain. Spontaneous rupture of HGB is rarely encountered, but it is possible to hypothesize that diabetic patients are more at risk since the size of the HGB persistently increases in this population even in the absence of an overt obstruction. Hence, patients with diabetic neuropathy primarily, gastroparesis should be evaluated for gallbladder dysfunction to avoid long-term complications in the future. Therefore, if HGB is uncovered in a diabetic patient, the traditional "watch-and-wait" approach may not apply to this highrisk population. Further research is warranted to determine if insulin-dependent diabetic patients are more prone to HGB than their non-insulin-dependent counterparts and if any promotility agents can play a role in the reversal of HGB.

\section{Acknowledgments}

None to declare.

\section{Financial Disclosure}

No financial disclosures or funding.

\section{Conflict of Interest}

None to declare. 


\section{Informed Consent}

We hereby confirm that informed consent was obtained from the patient for publication of the case details.

\section{Author Contributions}

Shivantha Amarnath contributed to the literature research and writing of manuscript. Abhishek Polavarapu contributed to the editing and reviewing of the manuscript. Vivek Gumaste was the study mentor and contributed to critical revision of the manuscript.

\section{References}

1. Whiting DR, Guariguata L, Weil C, Shaw J. IDF diabetes atlas: global estimates of the prevalence of diabetes for 2011 and 2030. Diabetes Res Clin Pract. 2011;94(3):311321.

2. Albu S, Voidazan S, Popa D. Gallbladder hydrops associated with an episode of acute liver toxicity in the adult: may it be considered a surgical emergency or not? 2016;1(2):180.

3. Hanna ACG. Essential surgical practice: higher surgical training in general surgery, 5th ed. CRC Press; 2015.

4. Ghouri YA, Mian I, Bhattacharjee G, Bhattacharjee MJJJotP. Hydropic gallbladder in three patients with poorly controlled diabetes mellitus: what constitutes optimal management? 2015;16(3):290-294.

5. Aune D, Vatten LJ. Diabetes mellitus and the risk of gallbladder disease: A systematic review and meta-analysis of prospective studies. J Diabetes Complications. 2016;30(2):368-373.

6. Ugbaja CA, Ayoola OO, Ikem RT, Idowu BM. Gall blad- der volume and contractility in type 2 diabetes mellitus. African Journal of Diabetes Medicine 2015;23(2):9-12.

7. Rai GS, Baghel VS, Rai T, Vyas MM. Gall bladder dysfunction in chronic diabetics (type 2): an ultrasonography based prospective study. J International Journal of Research in Medical Sciences. 2016;4(2):8.

8. Khothsymuong RR, Kaminski J. Gallbladder Hydrops. Journal of Diagnostic Medical Sonography 2004;20(4):256-259.

9. Jones MW, Deppen JG. Gallbladder mucocele. In: StatPearls. Treasure Island (FL). 2018.

10. Arshad F, Laway BA, Rather TA, Kuchay MS, Khan SH. Impaired gallbladder motility in adults with newly detected type 2 diabetes and lack of reversibility after achieving euglycemia. Can J Diabetes. 2015;39(2):101-104.

11. Guliter S, Yilmaz S, Karakan T. Evaluation of gallbladder volume and motility in non-insulin-dependent diabetes mellitus patients using real-time ultrasonography. J Clin Gastroenterol. 2003;37(4):288-291.

12. Wilson IR, Hurrell MA, Pattinson NR, Chapman BA. The effect of simvastatin and bezafibrate on bile composition and gall-bladder emptying in female non-insulin-dependent diabetics. J Gastroenterol Hepatol. 1994;9(5):447451.

13. Derici H, Kamer E, Kara C, Unalp HR, Tansug T, Bozdag AD, Nazli O. Gallbladder perforation: clinical presentation, predisposing factors, and surgical outcomes of 46 patients. Turk J Gastroenterol. 2011;22(5):505-512.

14. Gunasekaran G, Naik D, Gupta A, Bhandari V, Kuppusamy M, Kumar G, Chishi NS. Gallbladder perforation: a single center experience of 32 cases. Korean J Hepatobiliary Pancreat Surg. 2015;19(1):6-10.

15. Agarwal AK, Miglani S, Singla S, Garg U, Dudeja RK, Goel A. Ultrasonographic evaluation of gallbladder volume in diabetics. J Assoc Physicians India. 2004;52:962965. 